\title{
Stochastic Volatility and Correction to the Heat Equation
}

\author{
Jean-Pierre Fouque, George Papanicolaou and Ronnie Sircar
}

\begin{abstract}
From a probabilist's point of view the Twentieth Century has been a century of Brownian motion with major inputs from - and applications to Economics and Physics starting with Bachelier's thesis in 1900 and Einstein's work in 1905. There has been a tremendous and rapidly increasing number of mathematical works on the Brownian motion and its many applications during the century, starting with Wiener's construction in 1923 and followed by the development of the stochastic calculus. It is only during the Sixties and early Seventies that it became an essential modelling tool in Economics with the works of Samuelson, Merton and the famous Black-Scholes formula for pricing options. The need for more complicated nonconstant volatility models in financial mathematics has been the motivation of numerous works during the Nineties. In particular a lot of attention has been paid to stochastic volatility models where the volatility is randomly fluctuating driven by an additional Brownian motion. The authors of this paper have shown that in presence of separation of time scales, between the main observed process and the volatility driving process, asymptotic methods are very efficient in capturing the effects of random volatility in simple universal corrections to constant volatility models. From the point of view of partial differential equations this method corresponds to a singular perturbation analysis. The aim of this paper is to recast this approach in the context of the heat equation and to propose a universal correction to its solution. This is an attempt to show that financial mathematics may also contribute to new ideas in Physics.
\end{abstract}

\section{Introduction}

Heat diffusion in a homogeneous medium is described by the following linear second order parabolic partial differential equation

$$
\frac{\partial u}{\partial t}=\kappa \Delta u,
$$

with an initial condition $u(0, x)=h(x)$ at time $t=0$, the space variable $x$ being in $\mathbb{R}^{d}$ and $h$ being a bounded smooth nonnegative function for instance. The positive constant $\kappa$ is the diffusion constant. For simplicity we first describe the main idea in the one-dimensional case $d=1$ to avoid dealing with diffusion matrices. 
In order to describe heat propagation in inhomogeneous media one can make $\kappa$ dependent on the position $x$ and eventually on time $t$. Under certain conditions on the varying coefficient $\kappa(t, x)$ the equation

$$
\frac{\partial u}{\partial t}=\kappa(t, x) \frac{\partial^{2} u}{\partial x^{2}}
$$

has a well-defined solution $u(t, x)$. One can also consider models where $\kappa(t, x)$ is random, leading to partial differential equations with random coefficients. In any case the solution $u$ is a complex nonlinear functional of the coefficient $\kappa$. In order to illustrate that, in the context of random coefficients, one can think of the problem of computing the "ensemble average" $\langle u\rangle$ which does not satisfy an obvious closed equation. In other words, in random or non random cases, the solution $u$ depends on the detailed structure of the coefficient $\kappa$. This poses the problem of modelling $\kappa$. There are situations where asymptotic theories can be used.

One example is the case of small fluctuations

$$
\kappa=\kappa_{0}+\varepsilon \kappa_{1}(t, x)
$$

where $\kappa_{0}$ is constant, $\kappa_{1}$ is varying (periodically, randomly,...) and $\varepsilon$ is a small positive parameter. Indeed it is be possible to expand $u$

$$
u=u_{0}+\varepsilon u_{1}+\mathcal{O}\left(\varepsilon^{2}\right),
$$

where $u_{0}$ solves the corresponding problem with the constant coefficient $\kappa_{0}$. The first correction $u_{1}$, given by

$$
\frac{\partial u_{1}}{\partial t}=\kappa_{0} \frac{\partial^{2} u_{1}}{\partial x^{2}}+\kappa_{1}(t, x) \frac{\partial^{2} u_{0}}{\partial x^{2}}
$$

with a zero initial condition, depends strongly on the details of the perturbation $\kappa_{1}$.

Another interesting situation corresponds to the case where $\kappa$ is varying (periodically, randomly, ...) on smaller scales than the original scale of observation of $u$. The small parameter $\varepsilon$ is now a scale ratio and homogenization techniques enable us to approximate the problem by a diffusion problem with a constant coefficient corresponding to an effective medium. The first correction will again depend on the details of the model. We refer to [1] for details on these techniques. To illustrate our point one can think of a coefficient $\kappa$ varying rapidly in time, modelled by

$$
\kappa^{\varepsilon}(t)=\kappa\left(\frac{t}{\varepsilon}\right) .
$$

For instance $\kappa(t)$ may be an ergodic process in which case our problem becomes an averaging problem. Under appropriate mixing conditions on $\kappa(t)$ one can show that the solution $u$ is approximated by the solution $u_{0}$ of the constant coefficient heat equation

$$
\frac{\partial u_{0}}{\partial t}=\bar{\kappa} \frac{\partial^{2} u_{0}}{\partial x^{2}}
$$


The initial condition is $u_{0}(0, x)=h(x)$, the constant $\bar{\kappa}$ is the mean of the invariant distribution of the process $\kappa(t)$ and the small fluctuations around $u_{0}$ are random and determined by details of the model.

From a probabilist's point of view one can introduce a "virtual particle" whose position is given by $X_{t}=x+\sigma W_{t}$ where $\left(W_{t}\right)$ is a standard Brownian motion and $\sigma=\sqrt{2 \kappa}$. The solution of the constant coefficient heat equation is given by

$$
u(t, x)=\mathbb{E}\left\{h\left(X_{t}\right) \mid X_{0}=x\right\} .
$$

This is actually a backward Kolmogorov equation and in what follows it will be more natural to rewrite it with respect to a backward time variable leading to the problem

$$
\frac{\partial u}{\partial t}+\kappa \frac{\partial^{2} u}{\partial x^{2}}=0
$$

with the terminal condition $u(T, x)=h(x)$ at a final time $T$. Its solution is given by

$$
u(t, x)=\mathbb{E}\left\{h\left(X_{T}\right) \mid X_{t}=x\right\},
$$

where $\left(X_{t}\right)$ is the diffusion process given by

$$
d X_{t}=\sigma d W_{t} .
$$

In derivative pricing problems $\sigma$ would be the volatility of a risky asset where $X_{t}$ denotes the logarithm of its price and where the drift term has been omitted.

Considering corresponding pricing problems in the context of stochastic volatility, one naturally replaces $u(t, x)$ by

$$
u(t, x, y)=\mathbb{E}\left\{h\left(X_{T}\right) \mid X_{t}=x, Y_{t}=y\right\},
$$

where

$$
d X_{t}=f\left(Y_{t}\right) d W_{t} .
$$

The function $f$ is positive, we assume $f$ and $1 / f$ bounded for instance, and the random volatility $\sigma_{t}=f\left(Y_{t}\right)$ is driven by an ergodic Markov process to reflect the "mean-reverting" nature of stochastic volatilities. The expectation in (5) is taken with respect to the Equivalent Martingale Measure chosen by the market. This means that $\left(Y_{t}\right)$ should reflect the so-called market price of volatility risk. We refer to [3] for a detailed presentation. An important class of models is obtained by considering diffusion processes $Y$ driven by Brownian motions correlated to the Brownian motion $W$ driving $X$. This correlation is extremely important in pricing problems since it accounts for the skew or "smile" in implied volatility. Indeed it could not have been introduced at the level of the heat equation but it becomes very natural at the level of the virtual Brownian particle which may have its diffusion coefficient varying randomly in time.

Our toy-model of process $Y$ is based on the most simple ergodic diffusion process, namely the Ornstein-Uhlenbeck process. It is described by the stochastic differential equation

$$
d Y_{t}=\alpha\left(m-Y_{t}\right) d t+\beta\left(\rho d W_{t}+\sqrt{1-\rho^{2}} d Z_{t}\right),
$$


where $\alpha$ and $\beta$ are positive parameters and $W$ and $Z$ are two independent standard Brownian motions in such a way that $\left(\rho W_{t}+\sqrt{1-\rho^{2}} Z_{t}\right)$ is a standard Brownian motion correlated to $W$ through the correlation coefficient $\rho,|\rho|<1$.

In derivative pricing problems there is an additional drift term $-\beta \Lambda\left(Y_{t}\right) d t$ in (7) which represents the market prices of risk and determines the equivalent probability under which the expectation is computed. It turns out that this term gives rise, in the asymptotics we will consider, to a simple correction to the constant effective diffusion coefficient without affecting the nature of the correction. For this reason we simply ignore that term.

To summarize we propose to consider $u(t, x, y)$, given by (5), as a perturbation of the solution $u(t, x)$ of the constant coefficient heat equation given by (4). The underlying stochastic processes $(X, Y)$ are given by $(6,7)$. The fact that $u(t, x)$ and $u(t, x, y)$ are close to each other will be justified in the regime

$$
\alpha \text { large and } \beta^{2} / 2 \alpha \text { of order one. }
$$

The quantity $1 / \alpha$ is the intrinsic time scale of $Y$, its correlation time, which means that we assume that the volatility is fast mean-reverting as it has been found on market data in [4]. Assuming

$$
\nu^{2}=\beta^{2} / 2 \alpha=\mathcal{O}(1),
$$

means that the long-run, or equilibrium, fluctuation size remains of order one as $\alpha$ becomes large since $\nu^{2}$ is precisely the variance of the Gaussian $\mathcal{N}\left(m, \nu^{2}\right)$ invariant distribution of the OU process $Y$.

The function $u(t, x, y)$ given by (5) is the solution to the partial differential equation

$$
\frac{\partial u}{\partial t}+\frac{1}{2} f(y)^{2} \frac{\partial^{2} u}{\partial x^{2}}+\rho \beta f(y) \frac{\partial^{2} u}{\partial x \partial y}+\frac{1}{2} \beta^{2} \frac{\partial^{2} u}{\partial y^{2}}+\alpha(m-y) \frac{\partial u}{\partial y}=0,
$$

with the terminal condition $u(T, x, y)=h(x)$.

In Section 2 we show that, in the asymptotics $\alpha$ large and $\nu^{2}$ of order one, $u(t, x, y)$ is close to the solution $u_{0}(t, x)$ of a constant coefficient heat equation

$$
\frac{\partial u_{0}}{\partial t}+\bar{\kappa} \frac{\partial^{2} u_{0}}{\partial x^{2}}=0
$$

with the same terminal condition $u_{0}(t, x)$. That is in this sense that $u$ is a perturbation of $u_{0}$. We then derive the first correction which is of order $1 / \sqrt{\alpha}$.

Surprisingly this first correction does not depend on $y$ and it is simply proportional to the third space derivative of $u_{0}$.

In Section 3 we illustrate the universality of this type of correction by discussing other Markovian and non Markovian models, the latter requiring a martingale approach to the perturbation result. 


\section{Asymptotics}

It is convenient to introduce the small parameter $\varepsilon>0$ defined by

$$
\varepsilon=1 / \alpha,
$$

so that for a fixed value of $\nu$ we have

$$
\beta=\frac{\nu \sqrt{2}}{\sqrt{\varepsilon}} .
$$

We introduce the operator notation

$$
\begin{aligned}
\mathcal{L}_{0} & =\nu^{2} \frac{\partial^{2}}{\partial y^{2}}+(m-y) \frac{\partial}{\partial y} \\
\mathcal{L}_{1} & =\sqrt{2} \rho \nu f(y) \frac{\partial^{2}}{\partial x \partial y}, \\
\mathcal{L}_{2} & =\frac{\partial}{\partial t}+\frac{1}{2} f(y)^{2} \frac{\partial^{2}}{\partial x^{2}}=\mathcal{L}_{H}(f(y)),
\end{aligned}
$$

where

- $\mathcal{L}_{0}$ is the OU infinitesimal generator,

- $\mathcal{L}_{1}$ contains the mixed partial derivative due to the correlation $\rho$ between the Brownian motion driving $Y$ and $W$,

- $\mathcal{L}_{2}$ is the heat operator also denoted by $\mathcal{L}_{H}(f(y))$ to indicate its diffusion constant.

The partial differential equation (8) becomes

$$
\left(\frac{1}{\varepsilon} \mathcal{L}_{0}+\frac{1}{\sqrt{\varepsilon}} \mathcal{L}_{1}+\mathcal{L}_{2}\right) u^{\varepsilon}=0,
$$

where the solution is denoted by $u^{\varepsilon}(t, x, y)$ and satisfies the terminal condition

$$
u^{\varepsilon}(T, x, y)=h(x) \text {. }
$$

With the assumptions made on the coefficients and the function $h$, this equation has a unique solution $u^{\varepsilon}$ for any given value of $\varepsilon>0$. In particular the positive function $f$ is assumed to be bounded away from zero to avoid degeneracy in the diffusion.

Equation (13) is written in such a way that the coefficient of the time derivative is of order one so that the singular nature of the perturbation is revealed in the "diverging" terms.

Using the computation introduced in [2] and detailed in [3] one can expand the solution

$$
u^{\varepsilon}=u_{0}+\sqrt{\varepsilon} u_{1}+\varepsilon u_{2}+\varepsilon \sqrt{\varepsilon} u_{3}+\cdots,
$$

and deduce successively that:

- $\mathcal{L}_{0} u_{0}=0$ which is satisfied by choosing $u_{0}=u_{0}(t, x)$ independent of $y$.

- $\mathcal{L}_{0} u_{1}+\mathcal{L}_{1} u_{0}=\mathcal{L}_{0} u_{1}=0$ which is satisfied by choosing $u_{1}=u_{1}(t, x)$ independent of $y$ as well. 
- $\mathcal{L}_{0} u_{2}+\mathcal{L}_{1} u_{1}+\mathcal{L}_{2} u_{0}=\mathcal{L}_{0} u_{2}+\mathcal{L}_{2} u_{0}=0$ is a Poisson equation in $u_{2}$ with respect to $\mathcal{L}_{0}$ in the $y$-variable. Its solvablity condition is $\left\langle\mathcal{L}_{2} u_{0}\right\rangle=0$ where the average $\langle\cdots\rangle$ is taken with respect to the $\mathcal{N}\left(m, \nu^{2}\right)$ invariant distribution of the OU process $Y$.

- Using (12) and the fact that $u_{0}$ does not depend on $y$ we deduce that $u_{0}$ is the solution of the heat equation (9) with the effective constant diffusion

$$
\bar{\kappa}=\frac{1}{2}\left\langle f^{2}\right\rangle,
$$

and the terminal condition $u_{0}(T, x)=h(x)$.

- The equation for $u_{2}$ becomes

$$
\mathcal{L}_{0} u_{2}=-\frac{1}{2}\left(f^{2}(y)-\left\langle f^{2}\right\rangle\right) \frac{\partial^{2} u_{0}}{\partial x^{2}},
$$

and $u_{2}$ can be written

$$
u_{2}(t, x, y)=-\frac{1}{2} \phi(y) \frac{\partial^{2} u_{0}}{\partial x^{2}}+c(t, x),
$$

where $\phi(y)$ is a solution of the Poisson equation

$$
\mathcal{L}_{0} \phi=f^{2}-\left\langle f^{2}\right\rangle,
$$

and $c(t, x)$ does not depend of $y$.

- The terms of order $\sqrt{\varepsilon}$ give the following Poisson equation in $u_{3}$

$$
\mathcal{L}_{0} u_{3}+\mathcal{L}_{1} u_{2}+\mathcal{L}_{2} u_{1}=0,
$$

which leads the solvability condition

$$
\left\langle\mathcal{L}_{2} u_{1}\right\rangle+\left\langle\mathcal{L}_{1} u_{2}\right\rangle=0 .
$$

- Using the fact that $u_{1}$ does not depend on $y$, the form of $u_{2}$ obtained in (14) and the fact that $\mathcal{L}_{1} c=0$, we deduce the following equation for $u_{1}(t, x)$

$$
\frac{\partial u_{1}}{\partial t}+\bar{\kappa} \frac{\partial^{2} u_{1}}{\partial x^{2}}-\frac{\rho \nu}{\sqrt{2}}\left\langle f(y) \frac{\partial^{2}}{\partial x \partial y}\left(\phi(y) \frac{\partial^{2} u_{0}}{\partial x^{2}}\right)\right\rangle=0,
$$

which simplifies to

$$
\frac{\partial u_{1}}{\partial t}+\bar{\kappa} \frac{\partial^{2} u_{1}}{\partial x^{2}}-\frac{\rho \nu}{\sqrt{2}}\left\langle f \phi^{\prime}\right\rangle \frac{\partial^{3} u_{0}}{\partial x^{3}}=0,
$$

to be solved with a zero terminal condition at $t=T$.

Observe that $u_{1}(t, x)$ solves the heat equation with the constant diffusion constant $\bar{\kappa}$, a zero terminal condition, and a source term given by $C_{3} \frac{\partial^{3} u_{0}}{\partial x^{3}}$ where the constant

$$
C_{3}=-\frac{\rho \nu}{\sqrt{2}}\left\langle f \phi^{\prime}\right\rangle
$$


is of order one and proportional to $\rho$. One can easily check that the unique solution to this equation is given explicitly by

$$
u_{1}(t, x)=C_{3}(T-t) \frac{\partial^{3} u_{0}}{\partial x^{3}} .
$$

Setting $c_{3}=\sqrt{\varepsilon} C_{3}$ we obtain the main result of this section:

Theorem 1. For $x, y$ and $t<T$ fixed we have:

$$
u^{\varepsilon}(t, x, y)-\left(u_{0}(t, x)+c_{3}(T-t) \frac{\partial^{3} u_{0}}{\partial x^{3}}\right)=\mathcal{O}(\varepsilon) .
$$

The proof consists of introducing the difference

$$
Z^{\varepsilon}=u_{0}+\sqrt{\varepsilon} u_{1}+\varepsilon u_{2}+\varepsilon \sqrt{\varepsilon} u_{3}-u^{\varepsilon},
$$

the operator

$$
\mathcal{L}^{\varepsilon}=\frac{1}{\varepsilon} \mathcal{L}_{0}+\frac{1}{\sqrt{\varepsilon}} \mathcal{L}_{1}+\mathcal{L}_{2}
$$

and using the definitions of $u_{0}, u_{1}, u_{2}$ and $u_{3}$ to write the equation

$$
\mathcal{L}^{\varepsilon} Z^{\varepsilon}=\varepsilon\left(\mathcal{L}_{1} u_{3}+\mathcal{L}_{2} u_{2}\right)+\varepsilon \sqrt{\varepsilon} \mathcal{L}_{2} u_{3},
$$

to be solved with the terminal condition

$$
Z^{\varepsilon}(T, x, y)=\varepsilon\left(u_{2}(T, x, y)+\sqrt{\varepsilon} u_{3}(T, x, y)\right) .
$$

Setting

$$
\begin{aligned}
F^{\varepsilon}(t, x, y) & =\mathcal{L}_{1} u_{3}(t, x, y)+\mathcal{L}_{2} u_{2}(t, x, y)+\sqrt{\varepsilon} \mathcal{L}_{2} u_{3}(t, x, y) \\
G^{\varepsilon}(x, y) & =u_{2}(T, x, y)+\sqrt{\varepsilon} u_{3}(T, x, y),
\end{aligned}
$$

one can write the probabilistic representation of $Z^{\varepsilon}$

$$
Z^{\varepsilon}(t, x, y)=\varepsilon \mathbb{E}\left\{G^{\varepsilon}\left(X_{T}^{\varepsilon}, Y_{T}^{\varepsilon}\right)-\int_{t}^{T} F^{\varepsilon}\left(s, X_{s}^{\varepsilon}, Y_{s}^{\varepsilon}\right) d s \mid X_{t}^{\varepsilon}=x, Y_{t}^{\varepsilon}=y\right\} .
$$

Under the smoothness and boundedness assumption on the function $h$ and the boundedness of $f(y)$, so that solutions of Poisson equations are at most linearly growing in $|y|, F^{\varepsilon}$ and $G^{\varepsilon}$ are bounded uniformly in $x$ and at most linearly growing in $|y|$ and the result follows. The last argument requires uniform bounds in $\epsilon$ of moments of the process $Y$ given in the following lemma for the exponential moments.

Lemma 1. There exists a constant $c(v, y)$ such that for any $t \leq s \leq T$ and $\varepsilon>0$ we have

$$
\mathbb{E}\left\{e^{v Y_{s}}\right\} \leq c(v, y) .
$$

Proof. The process $Y$ is an OU process converging exponentially fast to its invariant distribution. Indeed $Y_{s}$ is Gaussian with mean $m+(y-m) e^{-(t-s) / \varepsilon}$ and variance $\nu^{2}\left(1-e^{-2(t-s) / \varepsilon}\right)$. Consequently

$$
\mathbb{E}\left\{e^{v Y_{s}}\right\}=\exp \left\{v\left(m+(y-m) e^{-(t-s) / \varepsilon}\right)+\frac{v^{2} \nu^{2}}{2}\left(1-e^{-2(t-s) / \varepsilon}\right)\right\}
$$


and the proof follows by choosing $c(v, y)=\exp \left\{|v|(|m|+|y-m|)+v^{2} \nu^{2} / 2\right\}$.

In Conclusion, a natural perturbation of $u_{0}$, the solution of the heat equation (9), is given by

$$
u_{0}+c_{3}(T-t) \frac{\partial^{3} u_{0}}{\partial x^{3}}
$$

where $c_{3}$ is a small parameter.

Setting $T-t=\tau$ and $\bar{\sigma}=\sqrt{2 \bar{\kappa}}, u_{0}$ is given by a convolution of $h$ with the Gaussian density

$$
g_{\bar{\sigma}^{2} \tau}(x)=\frac{1}{\sqrt{2 \pi \bar{\sigma}^{2} \tau}} e^{-\frac{x^{2}}{2 \bar{\sigma}^{2} \tau}},
$$

and the corrected value (18) is given by a convolution of $h$ with

$$
g_{\bar{\sigma}^{2} \tau}+c_{3} \tau \frac{\partial^{3} g_{\bar{\sigma}^{2} \tau}}{\partial x^{3}}
$$

which remains positive for all values of $x$ when $c_{3}$ is chosen negative and small enough as can be seen by a direct computation. This is illustrated in Figure 1. The shape of the corrected density has been observed on densities obtained from real financial data (see for instance [5]).

\section{Universality of the Correction}

The universality of the correction (17) resides in the fact that a large class of processes $\left(Y_{t}\right)$ and functions $f(y)$ will give rise to the same form, the only dependence on a particular choice being reflected in the value of the small parameter $c_{3}$. In our analysis the only required features of the process $Y$ are:

1. It has a diffusion part driven by a Brownian motion correlated to the Brownian motion driving the main process $X$.

2. It is ergodic and "running" on a fast time scale measured by its mixing rate.

3. It is mixing enough so that the corrector $\phi(y)$ introduce in (15) is smooth and at most polynomially growing at infinity.

4. In fact the process $Y$ does not need to be Markovian. We refer to ([3], Chapter 10) for generalizations to non-Markovian and multi-dimensional models and for a martingale approach to the derivation of the correction.

\section{References}

[1] A. Bensoussan, J. L. Lions and G. Papanicolaou Asymptotic analysis for periodic structures. Amsterdam, North Holland, 1978.

[2] J. P. Fouque, G. Papanicolaou and R. Sircar. Asymptotics of a Two-Scale Stochastic Volatility Model. In Equations aux derivées partielles et applications, Articles dediés à Jacques-Louis Lions, Gauthier-Villars, Paris (1998) 517-526.

[3] J. P. Fouque, G. Papanicolaou, and K. R. Sircar. Derivatives in Financial Markets with Stochastic Volatility. Cambridge University Press, 2000. 


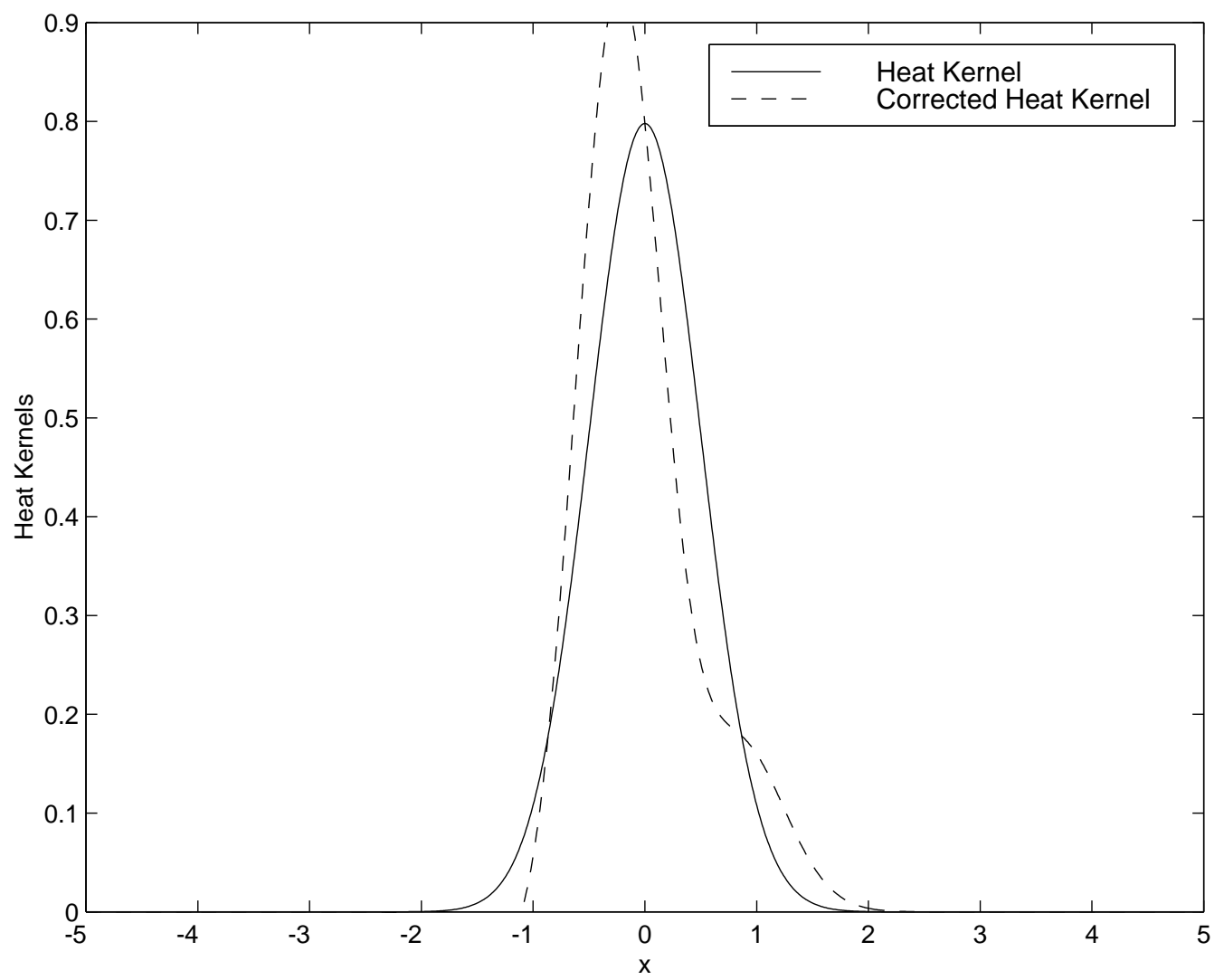

FIGURE 1. Gaussian heat kernel $g_{\bar{\sigma}^{2} \tau}$ and corrected heat kernel

with $\tau=1, \bar{\sigma}=0.5, c_{3}=-0.03$.

$$
g_{\bar{\sigma}^{2} \tau}+c_{3} \tau \frac{\partial^{3} g_{\bar{\sigma}^{2} \tau}}{\partial x^{3}}
$$

[4] J. P. Fouque, G. Papanicolaou, K. R. Sircar and K. Solna. Short time-scales in S\&P 500 volatility. To appear in the Journal of Computational Finance, 2003.

[5] Y. Li. A new algorithm for constructing implied binomial trees: does the implied model fit any volatility smile? Journal of Computational Finance, 4(2), Winter $2000 / 2001$.

Jean-Pierre Fouque,

Department of Mathematics,

North Carolina State University,

Raleigh NC 27695-8205, USA

Partially supported by NSF/DMS-0071744

E-mail address: fouque@math.ncsu.edu 
George Papanicolaou,

Department of Mathematics,

Stanford University,

Stanford CA 94305, USA

E-mail address: papanico@math.stanford.edu

Ronnie Sircar,

Department of Operations Research \& Financial Engineering, Princeton University, E-Quad

Princeton NJ 08544, USA

Partially supported by NSF/DMS-0090067

E-mail address: sircar@princeton.edu 